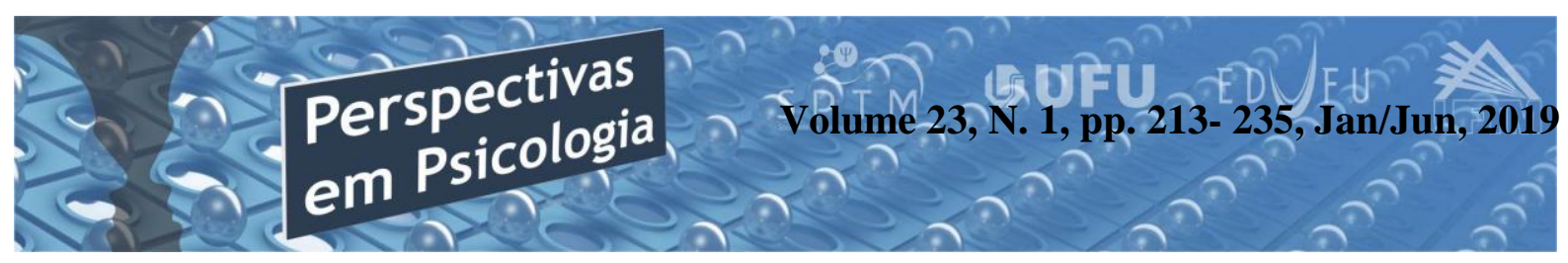

\title{
ATITUDES DE PROFISSIONAIS DE SAÚDE EM CUIDADOS PALIATIVOS SOBRE A MORTE E O MORRER
}

\author{
Letícia Dayane Moreira Silva \\ Marineia Crosara de Resende \\ Raphael Zardini Andrade \\ (Universidade Federal de Uberlândia - UFU - Uberlândia- MG)
}

\begin{abstract}
Resumo
Esse estudo teve como objetivos investigar as atitudes acerca da morte e do morrer de profissionais de saúde envolvidos nos cuidados paliativos de pacientes internados na UTIAdulto de um Hospital Universitário e correlacionar as atitudes em relação à morte/morrer com os dados profissionais/pessoais. Método: Participaram 20 profissionais de saúde (idade média 35 anos). Responderam aos instrumentos: a) ficha de dados sociodemográficos, b) questionário de dados profissionais e sobre o trabalho em cuidados paliativos, c) Escala de Avaliação do Perfil de Atitudes Acerca da Morte. Os Resultados indicaram que: os profissionais apresentaram atitude positiva de aceitação neutral, atitudes negativas em relação à aceitação da morte como escape, como evitamento, medo, como aceitação como aproximação. Houve correlação: quanto maior o medo da morte mais evitamento e menor a aceitação neutral. Quanto maior o evitamento, menor é a aceitação neutral. Quanto maior é aceitação neutral, menor é a aceitação como escape; menor a idade. As comparações indicaram diferença significativa para: estado civil (maior escore de aceitação de escape nos sem companheiro) e formação acadêmica (maior escore de evitamento nos com curso técnico). Os resultados obtidos podem ser reflexos da participação desses profissionais de saúde na criação do Serviço de Cuidados Paliativos no local em que atuam.
\end{abstract}

Palavras chave: atitudes frente a morte, cuidados paliativos, morte.

\section{Abstract \\ Attitudes on death and dying of health professionals in palliative care}

The present study aimed to investigate the attitudes about death and dying of health professionals involved in the palliative care of patients hospitalized in the ICU of a University Hospital and to correlate the attitudes regarding death between the scale and the professional/personal data. Method: Twenty health professionals working in the Adult ICU of the HC-UFU, with an average age of 35, took part. The following instruments were answered: a) socio-demographic data sheet, b) questionnaire of professional data and palliative care work, c) Revised Death Attitude Profile. Results: The professionals who work in palliative care in the Adult ICU presented a positive attitude of neutral acceptance (mean 5.51, SD = 0.87), negative attitudes regarding acceptance of death as escape (mean 2.60, SD = 1.32), as avoidance (mean 3.59, SD = 1.71), fear (mean 3.74, $\mathrm{SD}=1.47$ ), as acceptance as 
approximation (mean 3.76, SD $=1.05$ ). From the Spearman correlation it is possible to verify that the higher the fear of death, the more avoidance $(r=0.877 ; p \leq 0.05)$ and the lower the neutral acceptance $(\mathrm{r}=-0.653)$. The higher the avoidance, the lower the neutral acceptance $(\mathrm{r}$ $=-0.584)$. The higher the neutral acceptance, the lower the acceptance as escape $(r=-0.539)$ and the lower the age $(\mathrm{r}=-0.539)$. The comparisons indicated a significant difference for: civil status (highest escape acceptance score in those with no companion) and academic training (higher avoidance score in those with technical course). The results obtained may reflect the participation of these health professionals in the creation of the Palliative Care Service in the place where they work.

Keywords: attitudes towards death, palliative care, death.

\section{Introdução}

A morte é reconhecida como um evento natural, universal e inevitável, entretanto a capacidade do homem de pensar em sua própria finitude é restrita (Costa, 1999 citado por Von Hohendorff, $\&$ Melo, 2009). O medo daquilo que não se conhece, a morte, é acentuado pelo mistério e pelas incertezas que a caracteriza (Caputo, 2008). Do século XX herdou-se uma forma peculiar de pensar essa questão conhecida como "negação coletiva da morte". Trata-se de um conjunto de crenças, opiniões, costumes e práticas sociais que predominam na cultura contemporânea, principalmente nas atuais sociedades de consumo, de acordo com as quais não parece adequado falar sobre a finitude da existência (Verdade, 2008).

Segundo Verdade (2008), os efeitos biológicos do morrer do indivíduo adoecido são dolorosos e desencadeiam sentimentos de impotência e angústia nas pessoas que vivenciam de perto esse processo. A autora ainda salienta que entre as reações daqueles que estão em contato direto com a terminalidade do outro e, consequentemente, com a deterioração da vida física, está a incapacidade de conviver com a partida de alguém próximo e o distanciamento daqueles que necessitam de cuidados, consolo e acolhimento.

Em função dessa dificuldade, transferiram a morte do indivíduo adoecido de sua casa para o hospital (Cordeiro, 2017). O espaço domiciliar para morrer, cercado de hábitos e afetos foi substituído pelo hospital, local de ciência, razão e técnica (Àries, 1977 citado por Lopes, 2011). Dentro desse cenário, a morte passa a ter um novo local para acontecer, o hospital e mais especificamente as Unidades de Terapia Intensiva (UTIs). Segundo Carvalho e Arantes (2008), a UTI é o setor do hospital que recebe os pacientes que apresentam riscos de agravos e mortalidade em função de doenças 
agudas e crônicas, também é responsável pelos pacientes que necessitam de monitoramento intenso das variáveis fisiológicas e dos sinais e sintomas físicos. Com os avanços tecnológicos e os suportes de manutenção à vida, as UTIs se configuram como lugares onde ocorrem cada vez mais o prolongamento da vida dos pacientes, a obstinação terapêutica, ocasionando um alto custo financeiro, social e psicológico para todos os envolvidos no processo (Cordeiro, 2017).

Levando em consideração que a maioria dos óbitos hospitalares ocorre dentro das UTIs e a necessidade imperiosa de se estabelecer limites entre a melhor qualidade de vida possível e o prolongamento desta, cada vez mais tem se tornado imprescindível a consolidação dos Cuidados Paliativos nessas unidades hospitalares (Silva, Pedreira, Santos, \& Faustino, 2013). De modo simplista, a filosofia de cuidados paliativos busca oferecer a pessoa em fim de vida e aos familiares uma assistência humanizada, primando pelo respeito e dignidade, maximizando o conforto e minimizando o sofrimento (Serra, 2012).

\section{Cuidados Paliativos}

O verbo Palliar, com sua origem nos termos em latim palliare, pallium, refere semanticamente ao ato de proteger, cobrir com capa, entretanto, corriqueiramente paliar é atrelado ao significado de remediar, revestir de falsa aparência, adiar ou protelar e ainda aliviar provisoriamente. Cuidados paliativos referem-se a uma filosofia do cuidar, que visam o bem-estar e a qualidade de vida do paciente e de sua família diante de uma doença ou da necessidade de ser submetido a algum tratamento, sem possibilidade de cura (Costa Filho, Gutierrez, \& Mesquita, 2008).

Maciel (2008) esclarece que os cuidados paliativos e os curativos podem ocorrer concomitantemente. $\mathrm{Na}$ fase de diagnóstico e tratamento da doença, os cuidados paliativos podem ser realizados por equipes que não sejam necessariamente especializadas. A autora ressalta que quando a doença avança e no momento em que os cuidados curativos perdem sua eficácia, os cuidados paliativos passam a ocupar um lugar central no cuidado havendo a necessidade de equipes profissionais treinadas e qualificadas, para controlar e lidar com sintomas que vão além dos biológicos.

Carvalho e Arantes (2008), por meio de um estudo que ilustrou o cenário do processo de morrer em cinco hospitais universitários norte-americanos, identificou vários problemas no manejo ao paciente em terminalidade, a saber: a) metade dos pacientes sentiram dor 
considerável na maior parte do tempo; b) comunicação ruim entre médicos e pacientes; c) $30 \%$ das famílias envolvidas no estudo consumiram suas economias financeiras no tratamento do seu familiar; d) metade das ordens de "não ressuscitar" só foram descritas nos últimos dias de vida; e) a hospitalização final para os pacientes ultrapassaram oito dias; f) insatisfação da família relacionada ao tratamento despendido ao paciente. A partir dos resultados do estudo acima, os autores propuseram uma intervenção promotora de um cuidado mais humano, que considerasse os seguintes aspectos: a) Aperfeiçoar as diretrizes avançadas de vida e a comunicação entre pacientes e equipe; b) Realizar pesquisas sobre as dificuldades em prestar atenção em cuidados paliativos; c) compreender melhor a cultura dos hospitais e de outras instituições de saúde e adequá-la para atender as preferências do paciente; d) estimular diálogos sobre o sentido e o lugar da morte e da posição apropriada do cuidado nesse processo. Nessa filosofia de cuidado, o paciente é atendido no alívio à sua dor e nos sintomas dolorosos ao mesmo tempo em que recebe suporte afetivo e psicológico para seus sofrimentos, ou seja, o objetivo é a busca da "boa morte", com dignidade e o mínimo possível de sofrimento, acrescentando vida aos dias e não mais dias à vida (Kovács, 2003).
No entanto, como pontuam Carvalho e Arantes (2008), muitos casos são encaminhados para as UTIs devido à incapacidade das equipes de cuidarem de pacientes terminais, em decorrência da má comunicação entre os envolvidos no processo ou ainda pela dificuldade de aceitação da morte pela da família, pelo paciente ou pelos próprios profissionais envolvidos, que com o avanço tecnológico, a obstinação terapêutica torna-se comum e inicia-se uma luta não mais pela vida, mas sim contra a morte.

$$
\text { Fischer (2007) relata que o }
$$
contexto do cuidado ao paciente está entremeado por conflitos constantes de vida e morte, frustrações e dores, e que os profissionais de saúde todos os dias estão frente a essas circunstancias que exigem um grande desprendimento e capacidade de suportar e lidar com tais questões. Falco, Falco, Nascimento, Rodrigues, Soares, Rosa e Dias (2012) chamam a atenção para o modo como os profissionais de saúde percebem e concebem a morte, buscam desenfreadamente o prolongamento da vida, o que revela, na verdade, uma tentativa de afastar o sentimento de fracasso e a visão de inimiga que a morte desperta.

\section{Atitudes em relação à morte}


Para Kovács (2003) há diferenças significativas entre uma pessoa comum e um profissional de saúde no que se refere aos modos de lidar com a morte. Para os profissionais de saúde, a morte faz parte do cotidiano, uma companheira de trabalho que os colocam frente ao temor de sua própria morte todos os dias. Sentimentos de impotência e receio de transmitir ao paciente seus próprios medos e angústias, mal estar e envolvimento emocional são algumas das dificuldades apontadas pelos profissionais de saúde ao lidar com paciente em iminência de morte (Martins, Alves, \& Godoy, 1999).

Vicense (2014) enfatiza que os profissionais de saúde ao optarem por tal carreira, não se tornam livres de sentimentos, percepções e crenças em relação a morte, não eliminam a mortalidade de suas vidas, mas ao contrário, passam a ter maior contato com mortes e processos de morrer. A autora assinala que por mais preparados que possam estar esses profissionais, nem sempre sentem-se familiarizados com a morte.

Em um estudo realizado por Espíndola e Macedo (1994), com profissionais de saúde de uma UTI de um hospital geral do Rio de Janeiro, revelou-se que o diagnóstico, o prognóstico e a idade do paciente interferem na forma como os profissionais de saúde vivenciaram a morte dos pacientes. $\mathrm{O}$ estudo apontou que momentos de morte presenciados pelos profissionais de saúde eram permeados por sentimentos de fracasso, frustração e sensação de vazio. Boemer, Rossi e Nastare (1989 citados por Martins et al., 1999), que esclarecem que em UTIs o pensar a morte e o morrer são mais difíceis quando comparado a outros contextos, pois a dinâmica de funcionamento não possibilita momentos de reflexão.

Para Nogueira, Oliveira e Pimentel (2006), em decorrência da dificuldade de compreender a morte como inerente à vida, muitos profissionais de saúde adotam comportamentos defensivos como a utilização de uma linguagem técnica de difícil entendimento para leigos, quando em contato com a família. Outra prática apontada por esses autores é a negação através da omissão, ou seja, passam a árdua tarefa de cuidar daquele caso para um colega, se abstendo de contato com os envolvidos na "bomba-relógio" e finalizam dizendo que a incompreensão do quadro ou da possibilidade de morte tanto pelo paciente quanto pelos familiares, bem como os sentimentos decorrentes nesse contexto são algumas das consequências dessa prática.

$$
\text { Atitudes e percepções dos }
$$
profissionais de saúde em relação à morte são também influenciadas pela impossibilidade natural de uma 
representação inconsciente desse evento (Kovásc, 2002). Caputo (2008) corrobora e diz que no cerne da negação da morte há uma tentativa de afastar ou evitar a condição de finitos, entretanto, atitudes relacionadas à negação não impedem que a condição de finitude se concretize, mas tornam-se entraves no processo de elaboração e enfrentamento dessa condição que é inevitável.

Para Moreira e Lisboa (2006), vários aspectos psicológicos reverberam nos profissionais de saúde ao lidarem com pacientes em iminência de morte, uma vez que ao se depararem com a finitude do outro, esses profissionais estão face a face com a sua própria possibilidade de morte, o que muitas vezes pode levar a um distanciamento do paciente como mecanismo de defesa, tornando o cuidado restrito ao corpo e destituído de singularidade. $\mathrm{O}$ discurso de não estar preparado para lidar com a morte foi uma resposta recorrente em um estudo realizado com profissionais de saúde por Kovásc (2011). O referido estudo buscou discutir a comunicação sobre a morte em instituições de saúde.

Uma substancial contribuição em relação à morte e aos cuidados ao paciente enfermo foi dada por Cecily Saunders na década de 60, ao escrever sobre a filosofia do cuidado do paciente terminal. Segundo a visão desta autora o cuidado deve ser embasado em dois elementos principais: alívio do sofrimento físico com o controle efetivo da dor e um olhar atento às outras dimensões de sofrimento, psicológico e espiritual, um tratamento que enfatiza o cuidado em vez da cura (Falco et al., 2012; Cordeiro, 2017).

A partir disso e também com base nos trabalhos e pesquisas de Elisabeth Kübler-Ross (2005) com pacientes terminais, vem crescendo, na área da saúde, o movimento de humanização da morte. A autora explica que trata-se de uma abordagem na qual se busca propiciar ao paciente terminal ou com diagnóstico de doença sem possibilidades de cura, tratamento digno que considere suas vontades, emoções e angústias diante da proximidade da morte. $\mathrm{O}$ movimento de humanização do processo de morrer tenta mudar a condição, no qual o atendimento dado aos órgãos da pessoa tem mais qualidade do que o que é propiciado ao dono deles (Biff \& Mamede, 2004).

A postura humana frente à morte passou por modificações ao longo do tempo, sendo influenciada, sobretudo pelo advento dos hospitais e das UTIs. Neste contexto, os cuidados ao paciente enfermo e a terminalidade foram institucionalizados, passando a ser articulados pelos ditos profissionais de saúde, que podem ser emocionalmente 
impactados pela experiência dos cuidados e pelo recorrente contato com a finitude.

Vale ressaltar que em fevereiro de 2015 foi criado o Serviço de Cuidados Paliativos na UTI Adulto do Hospital de Clínicas, onde ocorreu a presente pesquisa, relatado por ( Silva Júnior, 2017), por meio do qual foram formados três grupos, com a finalidade de promover encontros de trabalho e estudos, em que os profissionais envolvidos deveriam discutir e definir quando incluir o paciente nos cuidados paliativos; estabelecerem melhorias na comunicação; ordenarem as melhores condutas com o paciente.

Pensando em conhecer as atitudes de profissionais que lidam com a morte e o morrer no seu dia a dia esta pesquisa teve como objetivos: (a) Investigar as atitudes acerca da morte e do morrer de profissionais de saúde envolvidos nos cuidados paliativos de pacientes internados na UTI de um Hospital Universitário. (b) Correlacionar as atitudes em relação à morte entre os escores da escala e com os dados profissionais/pessoais.

\section{Método}

\section{Participantes}

Participaram 20 profissionais de saúde que trabalham na UTI Adulto do HC-UFU, entre 22 e 55 anos (média 35; $\mathrm{DP}=7,75)$, a maioria entre 30 e 39 anos $(65,0 \%)$, do sexo feminino $(80,0 \%)$, casado $(50,0 \%)$, não tem filhos $(55,0 \%)$, mora com cônjuge $(45,0 \%)$ ou familiar $(30,0 \%)$, da religião católica $(65,0 \%)$, da área de enfermagem: técnicos $(35,0 \%)$ ou graduados $(15,0 \%)$. Na Tabela 1 é possível verificar as características sociodemográficas.

Tabela 1: Distribuição de frequência das características sociodemográficas dos participantes $(n=20)$.

\begin{tabular}{|c|c|c|}
\hline Variável & Porcentagem & Frequência \\
\hline \multicolumn{3}{|l|}{ Idade } \\
\hline $20-29$ & 4,0 & 4 \\
\hline $30-39$ & 65,0 & 13 \\
\hline$>-39$ & 15,0 & 3 \\
\hline \multicolumn{3}{|l|}{ Gênero } \\
\hline Masculino & 20,0 & 4 \\
\hline Feminino & 80,0 & 16 \\
\hline Total & 100,0 & 20 \\
\hline \multicolumn{3}{|l|}{ Estado Civil } \\
\hline Solteiro & 30,0 & 6 \\
\hline Casado & 50,0 & 10 \\
\hline Divorciado & 15,0 & 3 \\
\hline Viúvo & 5,0 & 1 \\
\hline
\end{tabular}




\begin{tabular}{lcc} 
Total & 100,0 & 20 \\
\hline Filhos & 55,0 & 11 \\
0 & 5,0 & 1 \\
1 & 35,0 & 7 \\
2 & 5 & 1 \\
3 & 100,0 & 20 \\
Total & & \\
Com quem mora & 25,0 & 5 \\
Sozinho & 30,0 & 6 \\
Familiar & 45,0 & 9 \\
Cônjuge & 100,0 & 20 \\
Total & & \\
Religião & 65,0 & 13 \\
Católica & 5,0 & 1 \\
Evangélica & 25,0 & 5 \\
Espírita & 5,0 & 1 \\
Sem religião & 100,0 & 20 \\
Total & & \\
\hline
\end{tabular}

\section{Profissão}

Assistente social $\quad 5,0$

Enfermeiro $\quad 15,0$

1

10,0

Fisioterapeuta

Médico

20,0

10,0

5,0

35,0

100,0

cuidados paliativos e $5 \%$ fizeram. Atuam

Formação profissional e atuação em cuidados paliativos

Com relação à formação profissional, $75,0 \%$ têm nível superior completo, sendo apenas graduação $(40,0 \%), \quad$ especialização $\quad(15,0 \%) \quad$ e mestrado (20,0\%). Tendo concluído sua formação há mais de 10 anos (50,0\%), entre 5 e 10 anos $(30,0 \%)$ e entre 1 e 5

anos $(20,0 \%)$. Dentre eles $95,0 \%$ não fizeram formação específica para atuar em na área intensivista há mais de 10 anos $(30,0 \%)$, entre 5 e 10 anos $(35,0 \%)$ e entre 1 e 5 anos $(35,0)$ e com cuidados paliativos há mais de 5 anos (31,6\%), entre 2 e 5 anos $(21,0 \%)$ e entre 1 e 2 anos $(47,4 \%)$.

Com relação à atuação específica em cuidados paliativos, $65,0 \%$ afirmam sentir-se preparados para trabalhar com cuidados paliativos e $35,0 \%$ afirmam não sentirem-se preparados. A respeito da efetividade da prática paliativa, $61,1 \%$ consideram a prática efetiva e $38,9 \%$ não acreditam na efetividade do serviço. Com relação à experiência pessoal, 65,0\% 
afirmaram nunca ter acompanhado um familiar e/ou ente em Cuidados Paliativos e 35,0\% já tiveram familiares/entes nessa situação. Entre os que já passaram pela experiência de acompanhar seu familiar/ente em cuidados paliativos, a avaliação quanto a qualidade do cuidado oferecido foi bom $(29,0 \%)$, regular (29,0\%), ruim $(14,0 \%)$, péssimo $(14,0 \%)$ e não se manifestaram $(14,0 \%)$.

\section{Instrumentos}

(a) Ficha de Informações sociodemográficas: Questionário para coleta de dados sobre idade, gênero, renda mensal, profissão, ocupação, estado civil e tipo de arranjo domiciliar.

(b) Dados profissionais e sobre o trabalho em cuidados paliativos: Questionário para coleta de dados acerca da formação profissional e atuação em cuidados paliativos e na área intensivista, o tempo de atuação em cuidados paliativos e na UTI. Questionou também se os profissionais se sentem preparados para trabalharem com cuidados paliativos e a avaliação que fazem dessa prática, bem como experiências pessoais sobre esse tema.

(c) Escala de Avaliação do Perfil de Atitudes Acerca da Morte (EAPAM) (Loureiro, 2010) - medida multidimensional para analisar a “aceitação da morte”, no último estágio do processo de morrer. Está organizado em 5 dimensões ou subescalas: aceitação neutral ou neutralidade (a morte é aceita como natural e sem receios), aceitação como aproximação (encarar a morte como um meio de passagem para uma vida feliz), aceitação como escape (morte encarada como um meio de fuga de uma existência com sofrimento), medo (para avaliar pensamentos e sentimentos negativos acerca da morte e processo de morrer) e evitamento (para avaliar atitudes de evitamento de pensamentos sobre a morte, de modo a diminuir o medo e ansiedade que os mesmos provocam).

A escala contém 32 itens, as dimensões ou subescalas são aferidas através de um número determinado de itens divididos nas cinco dimensões: a aceitação neutral e a aceitação como escape são aferidas através de 5 itens, a aceitação como aproximação é aferida através de 10 itens, a dimensão medo da morte é aferida através de 7 itens e a dimensão evitamento é aferida por 5 itens. É apresentada em forma de autorrelato escrito, em uma Estrutura Likert de 1 (Discordo totalmente) a 7 (Concordo totalmente).

$\mathrm{Na}$ Tabela 2 é possível verificar coeficientes de consistência interna ( $\alpha$ de Cronbach) para medir a confiabilidade da escala EAPAM. Verificou-se alta consistência interna $(\alpha>0,70)$ para a 
escala, exceto para a dimensão aceitação neutral $(\alpha=0,63)$. Os valores aqui encontrados são muito parecidos ao estudo de fidelidade do instrumento original, que apresentou valores de consistência interna razoáveis para cada uma das dimensões (todos acima de 0,80), à exceção de valor do coeficiente da dimensão "Aceitação neutral", que foi considerado modesto $(\alpha=0,64)$ (Wong, Reker, \& Gesser, 1994 citado por Loureiro, 2010).

Tabela 2 - Análise de consistência interna da escala EAPAM $(n=20)$.

\begin{tabular}{|c|c|}
\hline $\begin{array}{l}\text { Coeficiente } \\
\quad \alpha \text { de } \\
\text { Cronbach }\end{array}$ & $\begin{array}{cc} & \text { Coeficiente } \\
\text { Correlação } & \alpha \\
\text { com o Total* } & \text { (após } \\
& \text { retirada)** }\end{array}$ \\
\hline $\mathrm{Med}$ & --- \\
\hline 5 & --- \\
\hline Aceitação neutral & $\begin{array}{c}\text { EAPAM6 } \\
\text { EAPAM17 }\end{array}$ \\
\hline 10 & EAPAM31 \\
\hline eitação de escape & --- \\
\hline $\begin{array}{l}\text { Para a coleta de dados: O } \\
\text { levantamento de dados foi realizado nas } \\
\text { dependências da UTI adulto HC-UFU, } \\
\text { com o devido cuidado para não interferir } \\
\text { na rotina de trabalho dos profissionais. Os } \\
\text { profissionais foram abordados na saída do } \\
\text { trabalho, onde foram informados sobre os } \\
\text { objetivos da pesquisa e convidados a } \\
\text { participar, esclarecidos sobre o caráter } \\
\text { voluntário da participação e sobre garantia } \\
\text { de sigilo dos dados individuais. Após cada }\end{array}$ & $\begin{array}{l}\text { línio, sem considerar o item no escore total. } \\
\text { utiva dos itens com menor consistência. } \\
\text { mesmos pudessem responder de acordo } \\
\text { com sua disponibilidade de horário e } \\
\text { posteriormente, recolhidos pela } \\
\text { pesquisadora. } \\
\text { Procedimentos para análise de } \\
\text { dados: Para descrever o perfil da amostra } \\
\text { segundo as variáveis em estudo, foram } \\
\text { feitas tabelas de frequência das variáveis } \\
\text { categóricas, com valores de frequência } \\
\text { absoluta (n) e percentual (\%) e estatísticas } \\
\text { descritivas das variáveis numéricas, com } \\
\text { valores de média, desvio padrão, valores } \\
\text { mínimos e máximos, mediana e quartis. } \\
\text { Para comparação das variáveis numéricas } \\
\text { entre os grupos de níveis de estresse foram } \\
\text { utilizados os testes de Mann-Whitney (para } \\
\text { duas categorias) e de Kruskal-Wallis (para } \\
\text { três ou mais categorias), devido ao }\end{array}$ \\
\hline
\end{tabular}


tamanho reduzido da amostra e à ausência de distribuição normal dos escores. Para analisar a relação entre as variáveis numéricas foi utilizado o coeficiente de correlação de Spearman. O nível de significância adotado para os testes estatísticos foi de $5 \%$, ou seja, $\mathrm{p}<0,05$.

Procedimentos éticos: O projeto foi aprovado pelo Comitê de Ética da Universidade Federal de Uberlândia (Parecer $\mathrm{n}^{\circ}$ 1.824.330). Os procedimentos do presente estudo seguiram as normas regulamentadoras de pesquisa com seres humanos do Conselho Nacional de Saúde (Resolução 466/2012). Todos os participantes receberam informações acerca dos objetivos da pesquisa, das atividades a serem desenvolvidas e de seus direitos, antes de assinarem o Termo de Consentimento Livre e Esclarecido. A identidade de todos os participantes foi mantida em sigilo.

\section{Resultados}

Atitudes acerca da morte e do morrer

Os profissionais que atuam em cuidados paliativos na UTI Adulto apresentaram atitude positiva de aceitação neutral (média 5,51; DP=0,87), ou seja, a morte é aceita como natural e sem receios; e atitudes negativas em relação à aceitação da morte como escape (média 2,60; $\mathrm{DP}=1,32$ ), ou seja, não acham que a morte seja encarada como um meio de fuga de uma existência com sofrimento; como evitamento (média 3,59; $\mathrm{DP}=1,71$ ), não evitam pensamentos sobre a morte, de modo a diminuir o medo e a ansiedade que os mesmos provocam; medo (média 3,74; $\mathrm{DP}=1,47)$, ou seja, não apresentam pensamentos e sentimentos negativos acerca da morte e processo de morrer; e como aceitação como aproximação (média 3,76; $\mathrm{DP}=1,05$ ), encaram a morte como um meio de passagem para uma vida feliz.

\section{Correlação entre Escores da Escala}

A partir da correlação de Spearman é possível verificar que quanto maior o medo da morte mais evitamento ( $r=0,877$; $\mathrm{p} \leq 0,05)$ e menor a aceitação neutral $(\mathrm{r}=$ 0,653), ou seja quanto maior o medo da morte mais evitam pensar sobre ela e assim diminuem o medo e a ansiedade que esses pensamentos provocam e menor a aceitação da morte como um processo natural. Quanto maior o evitamento, menor é a aceitação neutral ( $\mathrm{r}=-0,584)$, ou seja, quanto mais evitam pensar sobre a morte, apresentam menos aceitação da morte como algo natural. Quanto maior é aceitação neutral, menor é a aceitação como escape $(r=-0,539)$ e menor a idade $(\mathrm{r}=-0,539)$, ou seja, quanto maior a aceitação da morte como algo natural, menos evitam pensar sobre ela.

Comparações dos escores da escala entre as variáveis categóricas 
A partir dos testes de MannWhitney (para comparação das variáveis entre 2 grupos) e de Kruskal-Wallis (para comparação das variáveis entre 3 ou mais grupos), verificou-se diferença significativa para: estado civil (maior escore de aceitação de escape nos sem companheiro), ou seja, aqueles que tem companheiros não encaram a morte como um meio de fuga de uma existência com sofrimento; e formação acadêmica (maior escore de evitamento nos com curso técnico), ou seja, os profissionais com formação técnica não evitam pensamentos sobre a morte. Ver Tabela 3.

Tabela 3 - Comparação dos escores da escala EAPAM entre variáveis categóricas.

\begin{tabular}{|c|c|c|c|c|c|c|c|c|c|c|}
\hline Estado Civil & VARIÁVEL & $\mathrm{N}$ & MÉDI & D. $\mathrm{P}$ & MI & Q1 & MEDIAN & Q3 & MÁ & VALOR \\
\hline \multirow[t]{5}{*}{$\mathrm{C} / \mathrm{COMP}$} & MedoMorte & 1 & 3,50 & 1,4 & 1,86 & 2,4 & 3,29 & 4,2 & $6 \Delta 2$ & D- \\
\hline & Evitamento & 1 & 3,32 & 1,7 & 1,00 & 2,0 & 2,90 & 5,0 & 6,00 & $\mathrm{P}=0,384$ \\
\hline & AceitacNeutr & 1 & 5,68 & 0,8 & 4,00 & 5,2 & 1,60 & 6,4 & 6,60 & $\mathrm{P}=0,403$ \\
\hline & AceitacRelig & 1 & 3,87 & 1,1 & 1,70 & 3,0 & 4,20 & 4,5 & 5,20 & $P=0,596$ \\
\hline & AceitacEscap & 1 & 2,04 & 1,1 & 1,00 & 1,2 & 1,60 & 2,6 & 4,80 & $\mathrm{P}=0,040$ \\
\hline \multirow[t]{5}{*}{ S/ COMP } & MedoMorte & 1 & 3,99 & 1,5 & 2,14 & 2,7 & 3,50 & 5,2 & 6,43 & \\
\hline & Evitamento & 1 & 3,86 & 1,7 & 1,20 & 2,6 & 3,60 & 5,6 & 6,80 & \\
\hline & AceitacNeutr & 1 & 5,34 & 0,9 & 3,80 & 4,4 & 5,40 & 6,0 & 6,80 & \\
\hline & AceitacRelig & 1 & 3,65 & 1,0 & 1,60 & 3,1 & 3,95 & 4,5 & 4,60 & \\
\hline & AceitacEscap & $\begin{array}{l}1 \\
n\end{array}$ & 3,16 & $\begin{array}{c}1,2 \\
6\end{array}$ & 1,00 & $\begin{array}{l}2,6 \\
n\end{array}$ & 3,20 & $\begin{array}{l}3,6 \\
n\end{array}$ & 5,60 & \\
\hline
\end{tabular}

\begin{tabular}{|c|c|c|c|c|c|c|c|c|c|c|}
\hline Formação & VARIÁVEL & $\mathrm{N}$ & MÉDI & D. $\mathrm{P}$ & MI & Q1 & MEDIAN & Q3 & MÁ & VALOR \\
\hline \multirow[t]{5}{*}{ FN/SI IPFR } & MedoMorte & 9 & 3,76 & 1,4 & 1,86 & 2,7 & 3,57 & 4,5 & 6,43 & $\mathrm{P}=$ \\
\hline & Evitamento & 9 & 3,69 & 1,5 & 1,80 & 2,4 & 3,80 & 5,0 & 6,00 & $P=$ \\
\hline & AceitacNeutr & 9 & 5,27 & 0,9 & 3,80 & 5,2 & 5,40 & 5,6 & 6,60 & $\mathrm{P}=$ \\
\hline & AceitacRelig & 9 & 4,07 & 0,60 & 3,00 & 3,9 & 4,20 & 4,5 & 4,90 & $\mathrm{P}=$ \\
\hline & AceitacEscap & 9 & 2,62 & 1,2 & 1,00 & 1,8 & 2,60 & 3,0 & 4,80 & $\mathrm{P}=$ \\
\hline \multirow[t]{5}{*}{ 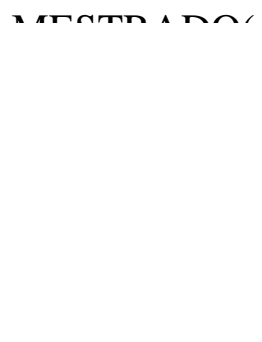 } & MedoMorte & 4 & 2,71 & 0,6 & 2,14 & 2,2 & 2,50 & 3,1 & 3,71 & \\
\hline & Evitamento & 4 & 2,30 & 1,0 & 1,00 & 1,5 & 2,40 & 3,1 & 3,40 & \\
\hline & AceitacNeutr & 4 & 6,25 & 0,3 & 5,80 & 6,0 & 6,30 & 6,5 & 6,60 & \\
\hline & AceitacRelig & 4 & 3,48 & 1,6 & 1,70 & 2,0 & 3,50 & 4,9 & 5,20 & \\
\hline & AceitacEscap & 4 & 1,75 & $\begin{array}{c}1,1 \\
1\end{array}$ & 1,00 & $\begin{array}{l}1,1 \\
n\end{array}$ & 1,30 & $\begin{array}{l}2,4 \\
n\end{array}$ & 3,40 & \\
\hline
\end{tabular}


ANDRADE

\begin{tabular}{|c|c|c|c|c|c|c|c|}
\hline & Mledolviorte & 4 & 5,39 & 1,2 & $3,71 \underset{\sim}{4,5}$ & 5,71 & $6,2 \quad 6,43$ \\
\hline & Evitamento & 4 & 5,60 & 0,9 & $4,40 \quad 5,0$ & 5,60 & $6,2 \quad 6,80$ \\
\hline & AceitacNeutr & 4 & 5,15 & 0,5 & $4,40 \quad 4,8$ & 5,20 & $5,5 \quad 5,80$ \\
\hline & AceitacRelig & 4 & 4,38 & 0,2 & $4,00 \quad 4,2$ & 4,45 & $4,5 \quad 4,60$ \\
\hline & AceitacEscap & 4 & 3,50 & $\begin{array}{c}1,5 \\
n\end{array}$ & $1,80 \underset{n}{2,4}$ & 3,30 & $\begin{array}{ll}4,6 \quad 5,60 \\
n\end{array}$ \\
\hline \multirow[t]{5}{*}{ ranared } & MedoMlorte & 3 & 2,86 & 0,6 & $2,142.1$ & 3,14 & $3,2 \quad 3,29$ \\
\hline & Evitamento & 3 & 2,33 & 1,1 & $1,201,2$ & 2,40 & $3,4 \quad 3,40$ \\
\hline & AceitacNeutr & 3 & 5,73 & 1,2 & $4,40 \quad 4,4$ & 6,00 & $6,8 \quad 6,80$ \\
\hline & AceitacRelig & 3 & 2,40 & 0,7 & $1,601,6$ & 2,50 & $3,1 \quad 3,10$ \\
\hline & AceitacEscap & 3 & 2,47 & 1,2 & $1,001,0$ & 3,00 & $3,4 \quad 3,4$ \\
\hline
\end{tabular}

* Valor-P referente ao teste de Mann-Whitney para comparação das variáveis entre 2 grupos.

** Valor-P referente ao teste de Kruskal-Wallis para comparação das variáveis entre 3 ou mais grupos.

\section{Discussão}

Os profissionais, que atuam em cuidados paliativos na UTI Adulto aqui pesquisada, acreditam que a morte é aceita como natural e sem receios; não acham que a morte seja encarada como um meio de fuga de uma existência com sofrimento; não evitam pensamentos sobre a morte, de modo a diminuir o medo e a ansiedade que os mesmos provocam; não apresentam pensamentos e sentimentos negativos acerca da morte e processo de morrer; e

encaram a morte como um meio de passagem para uma vida feliz. Loureiro

(2010) pontua que pessoas que apresentam atitudes positivas em relação à morte, não a temem e nem a desejam, por compreenderem que esta é inevitável e faz parte da vida, procuram conviver com a noção de mortalidade de modo positivo.

Para Lopes (2010), a aceitação da morte é adaptativa, uma vez que pessoas que aceitam a morte como inevitável fazem melhor uso da vida. A perspectiva de aceitação positiva da morte enfatiza que o processo de morrer e a morte é um interdito social, e quando é negativo passa a ser fator de ansiedade para os profissionais da saúde, que se sentem fracassados devido a não aceitação da morte como um evento natural (Vicense, 2014).

Os dados desta pesquisa mostraram que quanto maior o medo da morte, mais os profissionais evitam pensar sobre ela e assim diminuem o medo e a ansiedade que esses pensamentos provocam e menos acreditam que é um processo natural. Loureiro (2010) pontua que o modelo de aceitação interfere diretamente na 
ansiedade e no medo da morte (pensamentos, sentimentos acerca da morte e do morrer), por outro lado implica também em um evitamento (de pensar ou falar, de modo a reduzir a ansiedade e o medo). Para o autor isso ocorre porque nesse contexto surge a necessidade de atribuir sentido e significado à vida. A forma com que cada um aceita ou teme a morte depende subjetivamente da capacidade do individuo de atribuir significados à sua vida e, sobretudo, de quais significados tem atribuído ao longo de sua vida (Lopes, 2010).

Se existe o medo da morte e evitase entrar em contato com questões relacionadas a ela, a aceitação é menos provável, pois, como afirma Loureiro (2010), nessa dimensão não se tem medo e nem se cobiça a morte, mas a aceita como fato da vida, aproveitando ao máximo a existência. No âmbito da saúde, com a cristalização das relações vivenciadas diante da morte, o hospital pode se tornar um local de trabalho difícil de suportar e de atividades árduas (Martins et al., 1999). Assim a morte e o morrer tornam-se processos ansiogênicos (Costa Filho at. al., 2008), vez que quando os profissionais não aceitam a morte como um fenômeno natural, buscam recursos avançados para curar o enfermo, mesmo quando a possibilidade não exista.
O estudo de Silva e Menezes (2015), sobre os profissionais de saúde intensivistas apontam que apesar de conviverem frequentemente com $\mathrm{o}$ processo de morrer, esses profissionais demonstram dificuldades em aceitar a morte. Sobretudo a morte inesperada de pacientes jovens, principalmente quando se trata de quadros clínicos estáveis e com possibilidade de alta. Lopes (2010), ao realizar um estudo com 42 cuidadores profissionais de cuidados paliativos, concluiu que maior experiência profissional leva a maior aceitação da morte enquanto os cuidadores profissionais menos experientes (1-3anos) apresentaram resultados mais elevados em relação ao medo e ao evitamento da morte.

Mello e Silva (2012) revelaram que médicos convivem com a morte com sentimentos de estranheza e não com familiaridade, e apesar de lidarem mais com a possibilidade da morte que os leigos, os médicos não encaram a morte com naturalidade, se refugiam na impessoalidade e não se sentem confortáveis ao tratar do assunto. As autoras revelam ainda que a negação e o não envolvimento com a situação de morte do paciente, na verdade configura-se com uma estratégia de enfrentamento para lidar com o desconforto, sentimento de fracasso e derrota. Para Hermes e Lamarca (2013), os médicos se distanciam dos pacientes 
terminais e muitos passam a tratar o paciente não mais como pessoa, mas como objeto, em virtude das dificuldades em lidar com a finitude humana.

Acadêmicos de medicina admitiram, em pesquisa de Azeredo, Rocha e Carvalho (2010), que quando a morte se apresenta, sentem uma sensação de frustração, sentimento de incapacidade, devido ao despreparo, já que no modelo médico a cura parece ser a única meta. A concepção cultural da responsabilidade médica quanto à cura exige que a possibilidade de morte seja afastada e negada, pois a ocorrência da morte significa, de acordo com essa concepção, uma falha, gerando, nessa desarmonia, sofrimento (Santos, Aiok, \& OliveiraCardoso, 2013).

Santos e Hormanez (2013), em pesquisa sobre as atitudes de docentes do ensino superior do curso de enfermagem, diante da morte, acreditam que a falta de preparo na formação acadêmica em relação à morte, perpassa também os docentes. A insegurança e o despreparo fazem com que os profissionais da educação, docentes, busquem se proteger da dor e do sofrimento, mantendo uma postura distante e tratando a morte de modo impessoal; adotam métodos didáticos que visam uma aprendizagem, por parte dos alunos, de questões estritamente ligadas ao alívio do desconforto físico, impossibilitando reflexões sobre a morte.

Nesse cenário os alunos, futuros enfermeiros, internalizam a crença da necessidade de conter sentimentos e apresentam postura indiferente ou fria diante da terminalidade, reflexo da dificuldade de manejo dos professores, que não conseguem utilizar estratégias defensivas mais amadurecidas (Santos \& Hormanez, 2013). No entanto, no estudo de Pinho e Barbosa (2010), docentes relatam que há um grande desinteresse por parte dos discentes em relação às atividades relacionadas à morte e que os mesmos demonstram inquietação, angústia e dormem em sala de aula, se envolvendo nas atividades apenas quando as mesmas assumem um caráter obrigatório. Para Oliveira, Brêtas e Yamagut (2007), os estudantes de enfermagem sentem medo de expressarem seus sentimentos diante dos pacientes, o que pode ser resultado da crença de que não serão afetados em uma situação de morte.

Serra (2012), ao citar estudo realizado por Saraiva (2009), com 83 enfermeiros, afirma que os anos de experiência profissional e a idade do paciente que falece influenciam a atitude do enfermeiro perante a morte, mesmo assim, a morte não se torna mais fácil de ser encarada pelos enfermeiros, em virtude do constante confronto vivenciado por 
esses profissionais. Para Gutierrez e Ciampone (2007), crenças religiosas interferem no modo como os profissionais da enfermagem aceitam a morte.

No estudo de Ferreira, Lira, Siqueira e Queiroz (2013) que procura identificar quais as percepções de 15 psicólogos que trabalham em hospitais quanto aos conhecimentos, às habilidades e às atitudes relacionadas à morte, $40,0 \%$ dos profissionais definiram a morte como uma etapa de um processo de continuidade para além da morte, o início de outra vida ou de uma vida eterna, $33,4 \%$ definiram a morte como o fim da vida, $13,3 \%$ tinham dúvidas em relação à própria conceituação de morte e $13,3 \%$ a definiram como um evento natural e necessário. Em relação ao sentimento de medo, 40,0\% afirma ter algum tipo de medo em relação à morte, entre eles o medo de morrer lentamente, ocasionando a dependência de cuidados; morrer com dor; morrer cedo demais ou sem realizações; $54,0 \%$ declara não ter medo da morte; e $6 \%$ afirmou temer a morte de pessoas próximas e o impacto de sua morte nos familiares. As autoras citam que a qualidade do trabalho com a morte está relacionada a capacidade dos profissionais de saúde em identificar crenças relacionadas ao processo de morrer.

Marques, Oliveira e Marães (2006), em pesquisa com fisioterapeutas, revelaram que existe uma relação entre o tempo de atuação desse profissional com a vivência da morte do paciente, quanto maior o tempo de atuação em hospitais mais positiva é a vivência da morte do paciente para o fisioterapeuta. O estudo citado aponta também que todos os entrevistados apresentaram mecanismos de defesa para enfrentar a morte do paciente, tentam não se lembrar do fato, fingem que está tudo bem, buscam enfrentar a morte oferecendo suporte aos familiares e ainda afirmam tentar vivenciar essas questões apenas no hospital e não levá-las para casa e evitam, assim, envolvimento com o paciente.

De modo geral, as pesquisas indicam maior presença de crenças e atitudes negativas dos profissionais da área da saúde em relação à morte, no entanto esta pesquisa encontrou justamente $\mathrm{o}$ oposto, nossos resultados mostram outra possibilidade, que é de aceitação da morte por profissionais que convivem com pacientes terminais. As atitudes em relação à morte, sem dúvida, mudaram ao longo do tempo, assim como as necessidades sociais e biológicas têm evoluído, pensar a morte envolve uma cultura com seus valores individuais e coletivos; a soma dos fatores biopsicossociais fez com que a morte assumisse novo significado com diferentes impactos (Mercado García et al., 2016). 
Embora em muitos locais o tema morte continue sendo rejeitado, evitado, mesmo em áreas onde é recorrente diariamente, por exemplo, na área de saúde (Mercado García et al., 2016), a atitude de não evitar pensamentos sobre a morte foi um dado desta pesquisa, particularmente em profissionais com a formação técnica, na área de enfermagem. Os cuidados oferecidos por técnicos de enfermagem na UTI parecem convergir com o que afirmam Beraldo, Almeida e Bochi (2015), quando pontuam que é notável que esses profissionais de saúde reconhecem a morte, de pacientes sem nenhuma perspectiva de cura, como terapêutica e afirmam ainda que esse reconhecimento surge como uma ferramenta que auxilia o profissional a lidar com os percalços que aparecem diante do evento morte.

Para Trigueiro, Labronice, Raimondo e Paganini (2010), a aceitação da morte em seu tempo certo é uma atitude observável em profissionais enfermeiros, além de compreenderem a morte como pertencente à vida, aspectos relacionados à própria prática, como por exemplo, a empatia, o acolhimento e o cuidado humanizado contribuem para a ocorrência desse tipo de atitude. Fernandes, Iglesias e Avellar (2009) descrevem que os técnicos de enfermagem concebem a morte como um evento natural e tentam se acostumar com a ocorrência de óbitos, porém apresentam dificuldades de presenciar mortes. Para as autoras a busca de familiarização com a morte, nada mais é que a criação de estratégias de enfrentamento para lidar com a sua ocorrência inescapável no hospital.

Lopes (2010) afirma que diante de vivências difíceis, a aceitação da morte é uma estratégia de enfrentamento que leva ao alívio da dor e de sofrimentos de suas vidas; não se trata da "bondade da morte", mas da "maldade da vida". Os profissionais desta pesquisa, que têm companheiros, não encaram a morte como um meio de fuga de uma existência com sofrimento. A relação com companheiro(a), sua funcionalidade e a intimidade ajudam na proteção à saúde dos cônjuges, o suporte social familiar parece ser fundamental para a emergência do bem-estar subjetivo e da qualidade de vida (Pereira \& Resende, 2014). O suporte social exerce um papel fundamental na promoção e manutenção da saúde física e mental, é fator contribuinte na diminuição do estresse e favorecimento dos mecanismos de enfrentamento diante de dificuldades encontradas no dia a dia (Biffi \& Mamede, 2004).

\section{Considerações Finais}

Apesar da morte e o morrer serem eventos inevitáveis, uma questão bastante 
discutida na literatura é o desinteresse e esquiva em relação a essa temática. Em contrapartida à maioria das pesquisas nesta área, os profissionais de saúde participantes da presente pesquisa, apresentaram atitude positiva de aceitação neutral, ou seja, aceitam a morte como um evento natural e sem receios. Os profissionais desta pesquisa não evitam pensamentos sobre a morte e não apresentam pensamentos e sentimentos negativos em relação à morte em função de medo.

A presente pesquisa possibilitou o conhecimento das atitudes dos profissionais de saúde em relação à morte e o morrer, porém é importante sublinhar que os resultados obtidos ilustram a realidade da amostra em questão, naquele momento, uma visão mais ampla sobre essa temática requer estudos mais aprofundados.

Ao levarmos em conta os resultados obtidos nesta pesquisa, podemos afirmar que as atitudes em relação à morte parecem ter mudado. Dessa forma ressalta-se a importância de pesquisas futuras acerca de tal questão, buscando inclusive conhecer o que tem influenciado tal mudança.

\section{Referências}

Azeredo, N. S. G., \& Rocha, C.F. (2010). O Enfrentamento da Morte e do Morrer na Formação de Acadêmicos de Medicina. Revista Brasileira de Educação Médica, 35, 37 43. doi: 10.1590/S0100-55022011000100006.

Beraldo, L. M., Almeida, D. V. D., \& Bocchi, S. C. M. (2015). From frustration to coping with caring for death by nurse technicians. Revista brasileira de enfermagem, 68, 10131019. doi: 10.1590/0034-7167.2015680603i.

Biffi, R. G., \& Mamede, M. V. (2004). Suporte social na reabilitação da mulher mastectomizada: o papel do parceiro sexual. Revista da Escola de Enfermagem da USP, 38, 262-269. doi: 10.1590/S0080-62342004000300004

Caputo, R. F. (2008). O homem e suas representações sobre a morte e o morrer: um percurso histórico. Revista Saber Acadêmico, 6, 73-80. 
Carvalho, R. T., \& Arantes, A. C. L. Q. (2008). UTI. In: R. T. Carvalho, \& A. C. L. Q. Arantes (Orgs). Cuidado Paliativo. (pp. 178-194). São Paulo: Conselho Regional de Medicina do Estado de São Paulo.

Cordeiro, F. R. (2017). O retorno ao domicílio em cuidados paliativos: interface dos cenários brasileiro e francês. Tese de Doutorado, Universidade Federal do Rio Grande do Sul, UFRGS, Brasil.

Costa Filho, R. C., Gutierrez, F. L. B., \& Mesquita, A. F. (2008). Como implementar cuidados paliativos de qualidade na unidade de terapia intensiva. Revista Brasileira Terapia Intensiva, 20, 88-92. doi: 10.1590/S0103-507X2008000100014.

Falco, H. T., Falco, D., Nascimento, T. G., Rodrigues, M. R., Soares, M. L. S., Rosa, J., \& Dias, M. A. R. (2012). Cuidados paliativos na Unidade de Terapia Intensiva: uma discussão. Enfermagem Revista,15(2), 191-201.

Fernandes, P. V., Iglesias, A., \& Avellar, L. Z. (2009). O técnico de enfermagem diante da morte: concepções de morte para técnicos de enfermagem em oncologia e suas implicações na rotina de trabalho e na vida cotidiana. Psicologia: teoria $e$ prática, 11(1), 142-152.

Ferreira, R. A., Lira, N. P. M., Siqueira, A. L. N., \& Queiroz, E. (2013). Percepções de psicólogos da saúde em relação aos conhecimentos, às habilidades e às atitudes diante da morte. Psicologia: teoria e prática, 15(1), 65-75.

Fischer, J. M. K. J. (2007). Manual de tanatologia. Curitiba: Conselho Regional de Psicologia do Paraná.

Hermes, H. R., \& Lamarca, I. C. A. (2013). Cuidados paliativos: uma abordagem a partir das categorias profissionais de saúde. Ciência \& Saúde Coletiva, 18, 2577-2588. doi:10.1590/S1413-81232013000900012.

Kovács, M. J. (2002). Morrer com dignidade. Em M. M. M. J Carvalho (Org.), Introdução à Psiconcologia. (pp. 263- 278). Campinas: Livro Pleno. 
Kovács, M. J. (2003). Educação para a morte: desafio na formação de profissionais de saúde e educação. São Paulo: Casa do Psicólogo.

Kovács, M. J. (2011). Instituições de Saúde e a morte: Do interdito à comunicação. Revista Psicologia, Ciência e Profissão, 31, 482-503. doi: 10.1590/S141498932011000300005

Klüber-Ross, E. (2005). Sobre a morte e o morrer (8a ed.). São Paulo: Martins Fontes.

Lopes, A. S. C. (2011). Cuidador Informal: sobrecarga e atitudes face à morte. Dissertação de Mestrado, Escola Superior de Saúde de Viseu, Viseu.

Lopes, T. P.A.V. (2010). Atitudes perante a morte e ansiedade e depressão em cuidadores profissionais de cuidados paliativos. Dissertação de Mestrado, Universidade de Lisboa, Lisboa.

Loureiro, L. (2010). Tradução e adaptação da versão revista da Escala de Avaliação do Perfil de Atitudes acerca da Morte (EAPAM). Referência III Série, 1, 101-108.

Maciel, M. G.S. (2008). Definições e princípios: cuidados paliativos. In: Reinaldo Ayer de Oliveira (Coord.). Cuidado Paliativo. (pp. 15-32). São Paulo: CREMESP-Conselho Regional de Medicina do Estado de São Paulo.

Marques, A. F., Oliveira, D. N., \& Marães, V. R. F. S. (2016). O fisioterapeuta e a morte do paciente no contexto hospitalar: uma abordagem fenomenológica. Revista Neurociências, 14(2), 17-22. doi:10.1590/S0104-59702011000500012.

Martins, E. L., Alves, R. N., \& Godoy, S. A. F. (1999). Reações e sentimentos do profissional de enfermagem diante da morte. Revista Brasileira de Enfermagem, 52, 105-117. doi: 10.1590/S0034-71671999000100012.

Mello, A. A. M., \& Silva, L. C. (2012). A estranheza do médico frente à morte: lidando com a angústia da condição humana. Revista da Abordagem Gestáltica, 18(1), 52-60. 
Mercado García, L. R., García Rillo, A., Arceo Guzmán, M. E., Pimentel Ramírez, M. L., Díaz Flores, M., \& Arauz Contreras, J. (2016). Actitud hacia la muerte y su relación con la empatía médica en estudiantes de Medicina. Educación Médica Superior, 30(1) Recuperado en 22 de febrero de 2017, de http://scielo.sld.cu/scielo.php?script=sci_arttext\&pid=S086421412016000100004\&lng= es\&tlng=es.

Moreira, A. D. C., \& Lisboa, M. T. L. (2006). A Morte-Entre o Público e o Privado: reflexões para a prática profissional de enfermagem. Revista Enfermagem. UERJ, 14(3), 447-454.

Nogueira, A. C. C., Oliveira, L. M., \& Pimentel, V. (2006). O Profissional da Saúde e a Finitude Humana - A negação da morte no cotidiano profissional da assistência hospitalar. Revista Virtual Textos \& Contextos, 5, 1-11. doi: 10.15448/1677-9509.

Oliveira, J. R., Brêtas, J. R. S., \& Yamaguti, L. (2007). A morte e o morrer segundo representações de estudantes de enfermagem. Revista da Escola de Enfermagem da USP, 41, 386-394. doi: 10.1590/S0080-62342007000300007.

Pereira, I. A., \& Resende, M. C. (2014). Suporte social e depressão em idosos com doença arterial coronariana. Perspectivas em Psicologia, 18, 97-111. doi: 10.1590/S0103$863 \times 2008000200006$.

Pinho, L. M. O., \& Barbosa, M. A. (2010). A relação docente-acadêmico no enfrentamento do morrer. Revista da Escola de Enfermagem da USP, 44, 107-112. doi: 10.1590/S008062342010000100015 .

Santos, M. A., \& Hormanez, M. (2013). Atitude frente à morte em profissionais e estudantes de enfermagem: revisão da produção científica da última década. Revista Ciência \& Saúde Coletiva, 18, 2757- 2768. doi: 10.1590/S1413-81232013000900031.

Santos, M. A., Aoki, F. C. O. S., \& Oliveira-Cardoso, E. A. (2013). Significado da morte para médicos frente à situação de terminalidade de pacientes submetidos ao Transplante de 
Medula Óssea. Ciência \& Saúde Coletiva, 18, 2625-2634. doi: 10.1590/S141381232013000900017.

Serra, S. S. S. (2012). Morrer na presença de quem cuida: Atitudes do enfermeiro de cuidados diferenciados face à morte e aos cuidados ao paciente em fim-de-vida. Dissertação de Mestrado, Curso de Medicina da Universidade do Porto, Porto.

Silva, C. F, Souza, D. M., Pedreira, L. C, Santos, M. R., \& Faustino, T. N. (2013). Concepções da equipe multiprofissional sobre a implementação dos cuidados paliativos na unidade de terapia intensiva. Ciência \& Saúde Coletiva, 18, 25972604. doi:10.1590/S1413-81232013000900014.

Silva, N. R., \& Menezes, A. R. (2015). "Se parar, parou”: Categorização do morrer em um unidade de terapia intensiva do Rio de Janeiro. Revista de Saúde Coletiva, 25, 265-285. doi: 10.1590/S0103-73312015000100015.

Silva Júnior, V. Implantação do Serviço de Cuidados Paliativos em uma UTI Adulto de um Hospital Escola. Trabalho de Conclusão de Residência (Especialização em Saúde), Programa de Residência Multiprofissional e em Área Profissional da Saúde. Faculdade de Medicina da Universidade Federal de Uberlândia.

Spíndola, T., \& Macedo, M. C. S. (1994). A morte no hospital e seu significado para os profissionais. Revista Brasileira de Enfermagem, 47(2), 108-117.

Trigueiro, T. H., Labronici, L. M., Raimondo, M. L., \& Paganini, M. C. (2011). Dilemas éticos vividos pelos enfermeiros diante da ordem de não reanimação. Ciência, Cuidado e Saúde, 9, 721-727. doi:104025/cienccuidsaudev9i413824.

Verdade, M. M. (2008). Ecologia mental da morte. Um novo olhar, uma nova escuta para a Psicologia da Morte. (pp 162-193). In: M. J. Kovács (org). Morte e Existência Humana. Rio de Janeiro: Guanabara Koogan.

Vicense, M. C. (2016). Reflexão sobre a morte e o morrer na UTI: a perspectiva do profissional. Revista Bioética, 24(1), 64-72. 
Von Hohendorff, J., \& Melo, W. V. (2009). Compreensão da morte e desenvolvimento Humano: contribuições à Psicologia Hospitalar. Estudos e Pesquisa em Psicologia, 9(2), 480-492.

\section{Os autores:}

Letícia Dayane Moreira Silva, Programa de Residência Multiprofissional e em área profissional da saúde, Faculdade de Medicina, Universidade Federal de Uberlândia, Uberlândia, Minas Gerais, Brasil. Endereço: Rua da Tulha, 2997, Bairro Minas Gerais- Uberlândia, Minas Gerais. Tel. 34991253019

Marineia Crosara de Resende (Orientadora), doutorado em Educação pela Universidade Estadual de Campinas, Brasil (2006), Professor Associado II da Universidade Federal de Uberlândia, Brasil Instituto de Psicologia da Universidade Federal de Uberlândia.

Raphael Zardini Andrade (Co-Orientador), mestrado em Ciências da Saúde pela Universidade Federal de Uberlândia, Brasil (2016). Psicólogo do Hospital de Clínicas de Uberlândia, Brasil Centro de Referência Nacional em Hanseníase e Dermatologia Sanitária HC/UFU.

Recebido em: 10/01/2019

Aprovado em: 30/06/2019 